\title{
PRESSURE PUMP POWER CONTROL IN THE PRIMARY CIRCUIT OF THE HEAT EXCHANGE SYSTEM
}

\author{
Aleksandr Shilin ${ }^{1, *}$, Viktor Bukreev ${ }^{1}$, and Sergey Prohorov ${ }^{1}$ \\ ${ }^{1}$ Tomsk Polytechnic University, 634050, Tomsk, Russia
}

\begin{abstract}
In this paper we consider the problem of speed in hot water systems where highly efficient plate heat exchanger is used. Especially marked the problem which is connected with long transition drive of constant speed exceeding the time of the heat exchanger accumulative tank emptying more than twice. As a regulating element in the heat exchange system there was proposed to use asynchronous electric drive of pressure pump in the primary circuit of the heat exchanger. For correct use of such electric drive we solved the problem of control object mathematical model synthesis, which has non-linear properties, in particular, the transfer coefficient of the circuit can vary in more than 6 times. At the same time there was revealed the dependence of the transfer coefficient on the motor speed, which must be considered in the controller synthesis. In conclusion we suggested the solutions of regulators synthesis tasks with customizable settings for speed and switchable structure between relay $\lambda$ and PI regulators.
\end{abstract}

\section{Introduction}

In hot water systems (HWS) as a control device the valve with a controlled rod position is traditionally used. The very position of the rod is set by driving the constant speed with three-point control [1]. The block diagram of such a system is shown in Fig. 1, where in addition to the valve we have a high-efficiency heat exchanger and circulation pumps in both primary circuits of flow. As a coolant in most cases ordinary water is used. The control of such systems is performed on programmable logic controllers (PLCs). The principle of hot water system control is as follows: the water temperature $T_{p d 3}$, which is supplied to the consumer, depends on the water temperature in the first circuit, which in turn depends on the ratio of the volume $q_{i}$ of the entering coolant from input to the volume $q_{l}$ circulating in the first circuit. In its turn the volume of the incoming coolant depends on the position of the controlled stem valve $h$, which is set by the drive. To use traditional PID controls it is enough to organize two circuits [2] the first one sets the valve position, the second one, including PID, calculates the specified position of the rod on the mismatch between the target $T_{\text {set }}$ and the measured temperature $T_{p d 2}$ values.

\footnotetext{
*Corresponding author: shilin@,tpu.ru
} 
In such a configuration it is possible to use ready-made libraries FBD of blocks [3] PLC producers [4], including the basic laws of dynamic objects control and self-tuning procedures of closed contours. It would seem that in this field there is no subject management problem, but with the advent of highly efficient plate heat exchanger there appeared the speed problem which has not been previously considered in heat consumption systems.

The use of modern automatic control theory in the field of optimal control for speed may give little effect in performance, which cannot solve the problem of hot water temperature dips at the time of a sharp change in consumption. Therefore, usually the system includes a storage tank, allowing to increase the inertia of the control object commensurate with the time of transition. However, it significantly increases the cost and size of the design of automatic hot water supply control.

The article explores a radical solution to increase the speed of HWS, where as the actuator pressure pump with frequency controlled asynchronous motor is used instead of the valve with a slow drive.

\section{Problem statement}

As the actuating element it is supposed to use frequency motor control of pressure pump included in the counter pressure drop of inlet and reverse coolant coming from the supplier. The block diagram and the mathematical model will have several basic differences that should be considered in the synthesis of a closed system. Therefore, for the implementation of the proposed control system it is necessary to perform the synthesis of a mathematical model, to determine the impact on the model and the structure of differences between the traditional models. To analyze the use of the traditional PID controller. To propose alternative methods to PID controller which can improve performance of the system as a whole. To perform numerical simulation and analysis of the results.

\section{The structure of the object and its mathematical model}

The mathematical model of the DHW control system with AC electric drive can be built using the methodology presented in [5], where the coolant mixing process is calculated using the equivalent circuit. In this case, the element of the structure mixing and the equivalent circuit can be represented as shown in Fig.1.

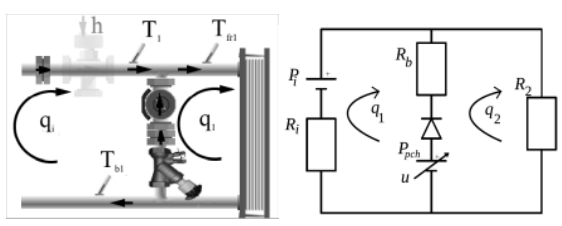

Fig. 1. Block diagram and equivalent circuit of coolant mixing in the frequency controlled drive.
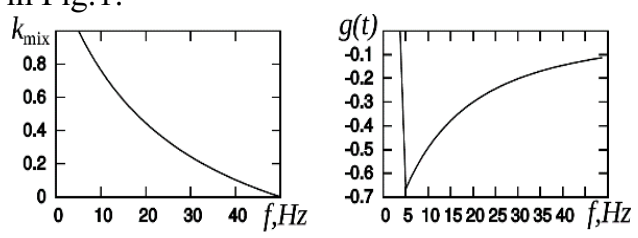

Fig. 2. Static dependence of mixing coefficient and transfer coefficient.

Kirchhoff equations will be as follows:

$$
\left\{\begin{array}{r}
P_{i}-P_{c h}(f)=q_{i}\left(R_{i}-R_{b}\right)-q_{2} R_{b} \\
P_{c h}(f)=q_{i}\left(R_{i}+R_{b}\right)-q_{i} R_{b}
\end{array}\right.
$$


where $\mathrm{P}_{\mathrm{i}}-$ the differential pressure generated by the central thermal point (CTP); $R_{i}-$ equivalent output hydraulic resistance of CTP; $P_{p c h}$ - pressure drop on the pressure pump; $R_{2}$ - hydraulic resistance of the heat exchanger input circuit; $R_{b}$ - hydraulic resistance of balancing valve.

It is required to find the mixing coefficient $k_{s m}(f)$ as a function depending on the converter frequency $f \in(5 ; 50)$. The result of calculating the coefficient of the mixing speed is a numerical solution of the nonlinear system of Kirchhoff's equations (1) on the characteristic points of the equilibrium state

From mixing characteristics, it can be seen (Fig. 2) that the control object has non-linear properties influencing on the transfer coefficient of the control object for different equilibrium points, which can be changed in six times. The presence of the equivalent output hydraulic resistance from the supplier point has a significant e ect on the nonlinear properties of the mixing coefficient. The pressure pump should be chosen so that it would be able to form a pressure difference $P_{p c h}$ greater than $P_{i}$ at least in three times.

To form a complete mathematical model of management we can use the results published in [5], the block diagram of which after inclusion frequency control differs from the traditional only in a fragment, shown in Fig. 1, a.

For the management method synthesis, it is advisable to use an equivalent model of the second order, which can be obtained using the methods presented in [6]. The procedure of obtaining a mathematical model of the second order is virtually not different, except the type of the nonlinear dependence of the mixing coefficient, which has now an inverse monotonic function of depending on the engine speed, so the model has a negative coefficient of the management transfer.

$$
\dot{x}_{1}=T_{0}^{-1}\left(g_{s m}\left(f_{p c h}\right) k_{u}(x, t) u_{s e t}(t-\tau)-x_{1}\right)
$$

where $\tau$ - equivalent delay of the elements with a short inertia, $T_{o}-$ inertia moment. The coefficient $k_{u}$ has a complex dependence on the parameters of the entire system and is presented in [6]. The transmission coefficient $g_{s m}$ has negative $g_{s m}<0$. This fact should be taken into account in the implementation of the system management.

When using the PID controller for a given system, there appear some problems associated with the specific application of the frequency converter and the limits on rate of change of differential pressure in the circuits of the plate heat exchanger. In particular, there is a limit to the rate of frequency change $f_{p L}$ and flux vector-clutch changing, which depend on the load on the motor shaft and other conditions [7-9].

In this case the component of the PD regulator suggests the possibility of an instantaneous change of the control voltage $u_{\text {set }}$ coming to the inverter input. The actual discrepancy between the actual shaft speed and a predetermined controller output make the restriction of the closed control system speed of the linear system on that should be considered when controller synthesis.

In this case more reliable equivalent mathematical model corresponds to the expression

$$
\left\{\begin{array}{l}
\cdot \\
x_{1}=\left(k_{\text {mix }}\left(x_{2}\right) k_{u}(x, t)-x_{1}\right) / T_{0} \\
\cdot \\
x_{1}=u(t-\tau) / T_{d r}
\end{array}\right.
$$

where $u(t)$ - the calculated control and the variable $x_{2}$ corresponds to $f_{p c h}$ on the condition that the time $T_{p r}$ exceeds the allowable time of rise speed and available for use in the control calculation. In this case the management $u(t)$ is imposed with limitation $(-1 ; 1)$. 


\section{Using the high-speed relay controller}

From the standpoint of high-speed optimality, it is advisable to use a -control [10], as its dynamic and optimal in performance properties are best suited to such systems [11]. It is possible to take advantage of softening condition of the control signal limiting. In the synthesis of -control the management is considered as three-position relay belonging to the set $(1 ; 0 ;+1)$, but in this case it is possible to use a continuous management value at the integrator input bounded with domain $[1:::+1]$. To assess the control quality there were chosen two methods of calculating control values implementation which were realized in $\mathrm{X}$-cos environment [4].

In the first case, $\lambda$-controller was used in the form given in the primary source [5] for the traditional HWS system in Fig. 4.

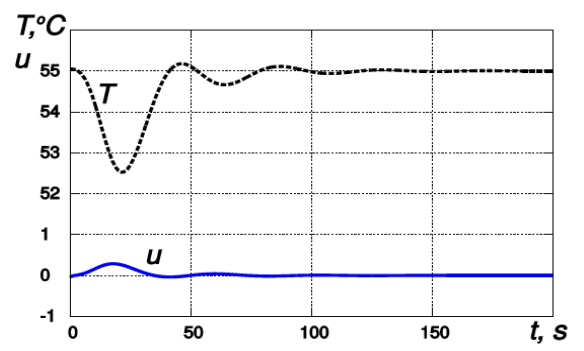

Fig 3. The transition process with the PI controller and frequency control.

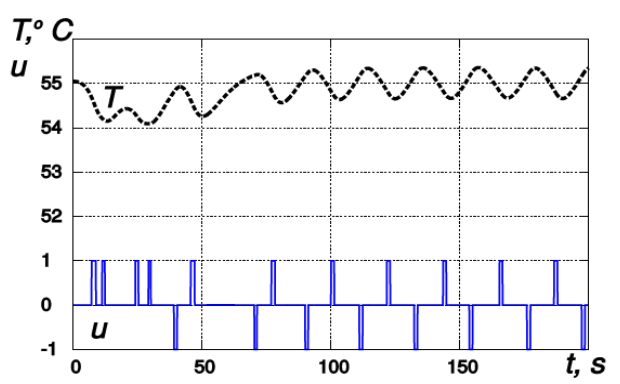

Fig. 4. The transition process with the $\lambda$ control.

The simulation results, where as the control element the drive frequency is taken, are given in Fig. 3 which shows that the temperature failure time is almost two times less than the simulation results, where a valve on drive with a constant speed is used.

Using $\lambda$-control in a frequency control system is shown in the Fig. 4 , where the temperature failure does not exceed one degree. The main disadvantage of this structure is the presence of minor fluctuations in the vicinity of the equilibrium point of the closed system. However, this disadvantage can be eliminated using a controller with a switchable structure, which forms the control in the vicinity of the equilibrium point by means of the linear regulator, built on the basis of the correction link with the transfer function. As a result, the obtained closed systems have the best speed for the given control object.

As an example and basis of further development we can consider PLC [4], built on the microprocessor STM32 of the series F1 [12], which allows the FBD-programming in a graphical environment and includes a control in its library. The microcontroller used in the PLC has a hard-tools [13] key control of the frequency converter for induction motor.

So, in presence of the means of FBD-programs compiling and the development of additional library item, which will perform the configuration of hard-means of frequency control, it is possible to create inexpensive hot water management system based on a microcontroller with significant functionality borrowed from the programming environment [13]. Constructive solutions are available in [7], and the necessary calculations of the power unit of the pump motor control can be taken both from [8] and [9].

\section{Conclusion}

In this paper we describe a fundamentally new approach to domestic hot water system management using electric drive; the direction of this research has become urgent in connection with a significant decline in prices for frequency converters. In the framework 
of the tasks there has been solved the problem of synthesis of a mathematical model suitable for research and development of asynchronous motor control methods.

It should be clarified that there are some unsolved scientific and practical problems in the way of further development. The function of transfer ratio of the engine speed is obtained from fairly simplified calculations to assess the range of variation of its value, taking into account mainly the principles

of operation of the system. At the same time the search for approximating functions could allow to consider the model of the object as deterministic, and there is a possibility to develop methods for the control synthesis with adjustable parameters in agreement with the current value of the calculated speed.

Using the principle of management with the switchable control structure between highspeed relay and linear asymptotically stable ones requires a systematic analysis of the stability of such systems. The obtained results of numerical experiments give evidence of such systems operability, therefore the search for solutions in the field of their analysis will provide valuable theoretical results.

\section{References}

1. A. Klyuev, A. Kolesnikov, Optimization of automatic speed control systems (Energoizdat, Moscow, 1982)

2. J. Shen, H. Chiang, 5th Asian Control Conference, 1, 472 (2004)

3. Scilab enterprises, open source software for numerical computation, (2015), http://www.scilab. Org

4. 3s-smart software solutions, the complete software suite for automation technology (2015), http://www.codesys.com

5. A. Shilin, V. Bukreev, Communications in Computer and Information Science, 487, 387 (2014)

6. A. Shilin, V. Bukreev, Thermal Engineering, 61, 741 (2014)

7. K. Buczek, J. Bartman, A. Koziorowska, Przeglad Elektrotechniczny, 83, 9 (2007)

8. A. Koziorowska, J. Bartman, International Journal of Numerical Modelling: Electronic Net-works, Devices and Fields, 25, 103 (2012)

9. M. Ioannides, IEEE Transactions on Energy Conversion, 19, 469 (2004)

10. A. Shilin, V. Bukreev, Devices and systems. Management, monitoring, diagnostics, Moscow, 10, 45 (2014)

11. L. Rozonoer, Autom. Remote Control, 64, 1237 (2003)

12. Stmicroelectronics, reference manual, stm32f103xx arm-based 32-bit mcus (2016), www.st.com/resource/en/reference_manual/cd00171190.pdf

13. Programmable logic controller west-02 (2015), http://npowest.ru/index.php/produktsiya/ programmiruemye-logicheskie-kontrollery 